\title{
A Putative Transcription Factor pcs1 Positively Regulates Both Conidiation and Sexual Reproduction in the Cereal Pathogen Fusarium graminearum
}

\author{
Boknam Jung ${ }^{1}$, Jungwook Park ${ }^{2}$, Hokyoung Son ${ }^{3}$, Yin-Won Lee ${ }^{3}$, Young-Su Seo ${ }^{2 *}$ and Jungkwan Lee ${ }^{1 *}$ \\ ${ }^{1}$ Department of Applied Biology, Dong-A University, Busan 604-714, Korea \\ ${ }^{2}$ Department of Microbiology, Pusan National University, Busan 609-735, Korea \\ ${ }^{3}$ Department of Agricultural Biotechnology and Center for Fungal Pathogenesis, Seoul National University, Seoul 151-921, \\ Korea
}

(Received on April 21, 2014; Revised on June 12, 2014; Accepted on June 12, 2014)

The plant pathogen Fusarium graminearum causes Fusarium head blight in cereal crops and produces mycotoxins that are harmful to animals and humans. For the initiation and spread of disease, asexual and sexual reproduction is required. Therefore, studies on fungal reproduction contribute to the development of new methods to control and maintain the fungal population. Screening a previously generated transcription factor mutant collection, we identified one putative $\mathrm{C}_{2} \mathrm{H}_{2}$ zincfinger transcription factor, pcs1, which is required for both sexual and asexual reproduction. Deleting pcs 1 in $F$. graminearum resulted in a dramatic reduction in conidial production and a complete loss of sexual reproduction. The pathways and gene ontology of pcs1dependent genes from microarray experiments showed that several G-protein related pathways, oxidase activity, ribosome biogenesis, and RNA binding and processing were highly enriched, suggesting that pcs 1 is involved in several different biological processes. Further, overexpression of $p c s 1$ increased conidial production and resulted in earlier maturation of ascospores compared to the wild-type strain. Additionally, the vegetative growth of the overexpression mutants was decreased in nutrient-rich conditions but was not different from the wild-type strain in nutrient-poor conditions. Overall, we discovered that the pcs 1 transcription factor positively regulates both conidiation and sexual reproduction and confers nutrient condition-dependent vegetative growth.

\footnotetext{
*Co-corresponding author.

J. Lee

Phone) +82-51-510-2267, FAX) +82-51-514-1778

E-mail: jungle@dau.ac.kr

Y.-S. Seo

Phone) +82-51-200-7554, FAX) +82-51-200-7505

E-mail)yseo2011@pusan.ac.kr
}

Keywords : Conidiation, Fusarium graminearum, Gibberella zeae, sexual reproduction, transcription factor

The plant pathogenic fungus Fusarium graminearum (teleomorph Gibberella zeae) causes Fusarium head blight in small grains and infected grains are frequently contaminated with mycotoxins, such as trichothecences and zearalenone (Leslie and Summerell, 2006). This fungus produces ascospores and conidia through sexual and asexual reproduction, respectively. Ascospores formed within perithecia are believed to be the primary inoculum of this disease, and conidia serve to disperse the disease (Fernando et al., 1997; Sutton, 1982).

$F$. graminearum is homothallic, but it can also cross with other strains in a heterothallic manner (Bowden and Leslie, 1999; Lee et al., 2003). In North America, high levels of $F$. graminearum genotypic diversity suggest that outcrossing often occurs in nature (Zeller et al., 2003, 2004). However, this may be a regional observation, as Korean populations show low genetic diversity (Lee et al., 2009, 2012). Many research groups have studied $F$. graminearum sexual reproduction because manipulation of the sexual stage is one of most efficient targets to decrease the primary inoculums and genetic diversity in field populations (Han et al., 2007; Hou et al., 2002; Lee et al., 2008, 2010; Son et al., 2011b).

Through asexual reproduction, $F$. graminearum produces macroconidia without microconidia, in contrast to many other Fusarium species that produce conidia with diverse shapes or sizes (Leslie and Summerell, 2006). A putative mitogen-activated protein kinase, Gpmk1, has been associated with conidial production (Zenczmionka et al., 2003), and gene expression profiles during conidial germination have been studied (Seong et al., 2008). However, despite the importance of conidia in disease dispersal, functional studies on conidiogenesis of $F$. graminearum are limited. 
F. graminearum undergoes vegetative growth and asexual reproduction in normal conditions, but sexual reproduction is only initiated under specific light and nutrient conditions. Because sexual reproduction requires many kinds of cellular differentiation, such as the development of antheridium, oogonium, crozier, ascus, and perithecia, asexual reproduction is blocked for F. graminearum in order to switch on sexual reproduction (Guenther and Trail, 2005; Hallen et al., 2007; Lee et al., 2010; Min et al., 2010; Qi et al., 2006; Yu et al., 2008). Recently, the genes for all annotated transcriptional factors were individually deleted and characterized for phenotypic changes (Son et al., 2011b), providing an in-depth study of each transcription factor responsible for specific developmental stages.

In this study, we screened the transcription factor deletion mutant library to identify genes related to both asexual and sexual reproduction. We identified a transcription factor named pcs 1 (positive regulator for conidiogenesis and sexual reproduction) and characterized the function of this gene by deletion, overexpression, and complementation. We found that pcs1-dependent genes are involved in basic biological processes, such as RNA processing and ribosome biogenesis, as well as in initial signaling cascades, such as signal receptors and G-protein coupled receptor signaling. Mutants with a $p c s 1$ deletion exhibited several important phenotypic changes, including differential vegetative growth, conidia germination, and sexual and asexual production. Specifically, we identified that $p c s 1$ positively regulates both asexual and sexual reproduction in $\mathrm{F}$. graminearum.

\section{Materials and Methods}

Fungal strains and media. The wild-type $F$. graminearum strain Z3639 (Bowden and Leslie, 1999) and mutants derived from it were stored as frozen conidial suspensions in $20 \%$ glycerol at $-70^{\circ} \mathrm{C}$. Fungal strains were maintained on potato dextrose agar (PDA), and the radial growth of strains was measured on PDA, minimal medium (MM), and complete medium (CM) (Leslie and Summerell, 2006). Sexual reproduction, including self, female, and male fertility, was induced on carrot agar as previously described (Lee et al., 2008) and carnation leaf agar (Leslie and Summerell, 2006). Asexual reproduction was induced both in carboxymethyl cellulose (CMC) (Capellini and Peterson, 1965) and on yeast malt agar (YMA) (Harris, 2005). Conidia germination was tested in MM and CM as previously described (Lee et al., 2009). All experiments were performed three

Table 1. Primers used in this study

\begin{tabular}{lll}
\hline Name & Sequence $\left(5^{\prime}-\right.$ - $\left.^{\prime}\right)$ & Reference \\
\hline Pcs-5F & AGTTGCTTGCTTCTCTGTCTATGTCTA & Son et al., 2011b \\
Pcs-5R & gcacaggtacacttgttagagCCACAATTGTAGGCTGGAGACC & Son et al., 2011b \\
Pcs-3F & cettcaatatcatttttgtcgCTTGCAAACAACACCAACACCACT & Son et al., 2011b \\
Pcs-3R & GCAATTGCGAAGATGGTAACT & Son et al., 2011b \\
Gen-F & CGACAGAAGATGATATTGAAGG & Min et al., 2010 \\
Gen-R & CTCTAAACAAGTGTACCTGTGC & Min et al., 2010 \\
Pcs-5N & TTTCCCCTTCTTTCTTTTTGGACCTAA & Son et al., 2011 \\
Pcs-3N & ATCCACCAGCGTCACATAACACAATAC & Son et al., 2011 \\
Pcs-rtF & CGCCCAGGACTTCTACGAGCATCT & This study \\
Pcs-rtR & CGGTGGTGGTAGGGAGGTTGTTCT & This study \\
Pcs-5R gfp & gaacagctcctcgccttgctcacCAGAGTGGTGTTGGTGTTGTTTGC & This study \\
Pcs-3F gfp & cctccactagctccagccaagccCTTGCAAACAACACCAACACCACT & This study \\
pIGPAPA-sGFP F & GTGAGCAAGGGCGAGGAGCTG & Son et al., 2011a \\
HYG-F1 & GGCTTGGCTGGAGCTAGTGGAGG & Son et al., 2011a \\
Pcs-5R OE & gatagtggaaaccgacgcccGAAGGGAGTGAAGCTTGTTGTGAATC & This study \\
Pcs-3F OE & cacaaaggaaccaatttcaagCATCATGACAGCCTTGTCAGATACG & This study \\
Pcs-3F gfp OE & cggcatggacgagctgtacaagATGACAGCCTTGTCAGATACGTCC & This study \\
Pcs-3R OE & TGGTGGAACGAATTGGCTGATACT & This study \\
Pcs-3N OE & TGGAAGTTGCTGGCCTGGAGAAAT & This study \\
Neo-for new & GGGGCGTCGGTTTCCACTATC & Lin et al., 2011 \\
EFpro-Rev & TTTGAAGATTGGGTTCCTTTTGTG & Lin et al., 2011 \\
eGFP-P1 & CTTGTACAGCTCGTCCATGCCG & Lin et al., 2011 \\
\hline
\end{tabular}


times with three replicate plates.

Nucleic acid manipulation and polymerase chain reaction (PCR). Fungal genomic DNA was extracted from $\mathrm{CM}$ culture as previously described (Leslie and Summerell, 2006). Total RNA was extracted from mycelia or conidia using an RNA-spin total RNA kit (Intron Biotech., Seongnam, Korea). First-strand cDNA was synthesized with SuperScriptIII reverse transcriptase (Invitrogen, Carlsbad, CA, USA). Quantitative real-time PCR (qRTPCR) was performed with SYBR Green Supermix (BioRad, Hercules, CA) and a 7500 real-time PCR system (Applied Biosystems). Elongation factor 1- $\beta$ (Broad Institute; FGSG_01008.3) was used as an endogenous control for normalization. The PCRs were repeated twice with two replicates per run. Standard procedures were used for restriction endonuclease digestion, DNA ligation, gel electrophoresis, and gel blotting (Sambrook and Russell, 2001). The PCR primers (Table 1) used in this study were synthesized by the Bioneer oligonucleotide synthesis facility (Bioneer Corp., Chungwon, Korea), diluted to $100 \mu \mathrm{M}$ in sterilized water, and stored at $-20^{\circ} \mathrm{C}$. General PCR reactions were performed as described previously (Lee et al., 2008).

Identification of pcs1 gene. A previous study identified 41 transcription factor mutants that had a dramatic reduction or no conidial production in CMC (Son et al., 2011b). Each strain was re-activated on PDA and inoculated both in CMC medium and YMA for conidial production as described above. In addition, each strain was tested for sexual reproduction on carnation leaf agar and carrot agar.

Complementation and overexpression of pcs1. To complement the $p c s 1$ deletion mutant, the $p c s 1$ open reading frame (ORF), including its promoter region and excluding its stop codon, was amplified from the wild-type strain of the genomic DNA of Z3639 with primers Pcs-5F and Pcs5R gfp. The GFP and hygromycin resistance gene cassette was amplified from pIGPAPA (Horwitz et al., 1999) with primers pIGPAPA-sGFP F and HYG-F1. This gene cassette was fused to the C-terminus of $p c s 1$ by double-joint PCR (Yu et al., 2004). The fused construct was amplified with primers Pcs-5N and Pcs-3N, and the amplicon was transformed into the $p c s 1$ deletion mutant. The phenotypes of the transformant were compared with those of the wildtype and pcs 1 deletion strains. Overexpression of pcs 1 was performed as previously described (Lin et al., 2011). Briefly, primer pairs Pcs-5F/Pcs-5R OE and Pcs-3F OE/ Pcs-3R OE were used to amplify the 5 ' flanking region of $p c s 1$ and the $p c s 1 \mathrm{ORF}$, respectively. A gen- $P_{E F I \alpha}$, carrying the elongation factor $1 \alpha$ promoter $\left(P_{E F I a}\right)$ from Fusarium verticillioides, was amplified from Pskgen (Lee et al., 2011) with primers Neo-for new/EFpro-Rev. The three amplicons were fused as described above. Using this fusion fragment as a template, a final PCR product was amplified with the nested primers Pcs-5N and Pcs-3N OE. Insertion of the construct was confirmed by Southern hybridization. The transcript level in the mutants was checked by realtime PCR with the Pcs-rtF/Pcs-rtR primer pair.

Fertility test. For the self-fertilization test, mycelia of strains grown on carrot agar for 5 to $7 \mathrm{~d}$ were mockfertilized, as previously described (Leslie and Summerell, 2006). For outcrossing tests, the heterothallic strain $\Delta$ mat1$1:: \mathrm{hH} 1::$ GFP (Hong et al., 2010), which carries a mat $1-1$ deletion and histone H1::GFP, was used as the female. The mutant strains generated in this study were used as males. Thus, all ascospore progeny resulted from these heterozygous crosses. Female mycelia on plates were fertilized with a suspension of conidia from the male strains grown on $\mathrm{CMC}$ medium, and the fertilized plates were incubated at $25^{\circ} \mathrm{C}$. Ascospores and asci in perithecia were observed 7 to $14 \mathrm{~d}$ after fertilization under the Carl Zeiss microscope DE/ Axio imager A1 (Carl Zeiss) equipped with a GFP filter (470/40 nm excitation, $495 \mathrm{~nm}$ dichroic, $525 / 50 \mathrm{~nm}$ emission). Images were analyzed with Axio vision software (Carl Zeiss).

cDNA synthesis and microarray analysis. Each strain was incubated in $50-\mathrm{ml} \mathrm{CM}$ for $72 \mathrm{~h}$ at $25^{\circ} \mathrm{C}$ on a rotary shaker $(150 \mathrm{rpm})$. Subsequently, mycelia were harvested and washed twice with 50-ml sterile water. Mycelia were spread on YMA to induce conidiation (Lee et al., 2009). After $48 \mathrm{~h}$, total RNA was extracted from cultures including mycelia and conidia. The cDNA synthesis and microarray hybridization were performed as previously described (Lee et al., 2010).

KEGG pathway and gene ontology (GO) enrichment analysis. All GO terms and KEGG pathway sources were downloaded using the Wget program and the direct downloading service of the web server from the Kyoto Encyclopedia of Genes and Genomes (KEGG, http://www.genome.jp/kegg/) and UniProt (http://www.uniprot.org/). In total, 1,280 pcs 1-dependent genes were annotated with data from both KEGG pathway and GO terms. Common results of annotated genes from both databases were enriched by Python coding script. All results of enriched KEGG pathways or GO terms were compared to the original genome 
according to the formula:

$$
P \text { value }=1-\sum_{\mathrm{x}=0}^{\mathrm{m}-1} \frac{\left(\begin{array}{c}
\mathrm{M} \\
\mathrm{x}
\end{array}\right)\left(\begin{array}{c}
\mathrm{N}-\mathrm{M} \\
\mathrm{n}-\mathrm{x}
\end{array}\right)}{\left(\begin{array}{l}
\mathrm{N} \\
\mathrm{n}
\end{array}\right)}
$$

where $\mathrm{N}$ is the number of genes with annotation by KEGG pathways or GO terms annotation; $\mathrm{n}$ is the number of pcs 1 dependent genes in $\mathrm{N}$; $\mathrm{M}$ is the number of genes annotated to the specific KEGG pathway or GO term; and $\mathrm{m}$ is the number of pcs1-dependent genes in $\mathrm{M}$. We chose only KEGG pathways and GO terms with a $P$ value of $<0.05$. To identify enriched GO gene categories, we classified all data by biological process, molecular function, and cellular component.

\section{Results}

Identification of pcs1. F. graminearum conidial development is suppressed during sexual reproduction; therefore, many genes related to sexual development work to suppress conidial production, and vice versa. However, we identified one deletion mutant that showed both dramatic reduction in conidial production and a complete loss in sexual development. The locus of this $F$. graminearum gene in the comparative Fusarium genome database is FGSG_01877.3, and it is annotated as a $\mathrm{C}_{2} \mathrm{H}_{2}$ zinc-finger transcription factor. The gene is 2881 bp with one intron, and the deduced amino acid size is 630 . This hypothetical protein is highly conserved among the Ascomycota, but the gene's function has not been studied. Based on the charac- teristics displayed by the deletion mutant, we designate this gene as pcsl.

pcs1 is required for sexual development. To confirm whether the phenotypic changes shown in pcs 1 deletion mutants were triggered by the deletion event of $p c s l$, the psc1 deletion mutant was complimented. The complimented strain was constructed by reintroducing the pcs 1 ORF, including its native promoter and terminator, into the deletion mutant (C-pcs1). In addition, to confirm the effect of $p s c 1$ overexpression, the construct with the pcs 1 ORF controlled under a constitutive promoter was introduced

Table 2. Mycelial growth and conidiation of Fusarium graminearum strains

\begin{tabular}{cccc}
\hline & \multicolumn{3}{c}{ Radial growth of mycelia $(\mathrm{mm})^{*}$} \\
\cline { 2 - 3 } & $\begin{array}{c}\text { Minimal } \\
\text { medium }\end{array}$ & $\begin{array}{c}\text { Complete } \\
\text { medium }\end{array}$ & $\begin{array}{c}\text { No. of conidia } \\
\left(\times 10^{5} / \mathrm{ml}\right)^{\S}\end{array}$ \\
\hline Z3639 & $76^{\mathrm{a}, \boldsymbol{f}}$ & $84^{\mathrm{a}}$ & $27^{\mathrm{b}}$ \\
D-pcs1-1 & $83^{\mathrm{b}}$ & $85^{\mathrm{a}}$ & $0.6^{\mathrm{a}}$ \\
D-pcs1-2 & $85^{\mathrm{b}}$ & $86^{\mathrm{a}}$ & $0.4^{\mathrm{a}}$ \\
C-pcs1-1 & $77^{\mathrm{a}}$ & $82^{\mathrm{a}}$ & $32^{\mathrm{b}}$ \\
C-pcs1-2 & $78^{\mathrm{a}}$ & $83^{\mathrm{a}}$ & $29^{\mathrm{b}}$ \\
O-pcs1-1 & $75^{\mathrm{a}}$ & $63^{\mathrm{b}}$ & $45^{\mathrm{c}}$ \\
O-pcs1-2 & $74^{\mathrm{a}}$ & $65^{\mathrm{b}}$ & $50^{\mathrm{c}}$ \\
\hline
\end{tabular}

*Each strain was grown on minimal medium and complete medium for 5 days af $25^{\circ} \mathrm{C}$ and radial growth was measured.

${ }^{\S}$ Fungal strains were grown in $50 \mathrm{ml}$ carboxymethyl cellulose medium for 5 days at $25^{\circ} \mathrm{C}$ in an orbital shaker $(150 \mathrm{rpm})$, and conidial production was measured using a hemocytometer.

"Values within a column not sharing a letter are significantly different according to Tukey's test $(P<0.05)$.
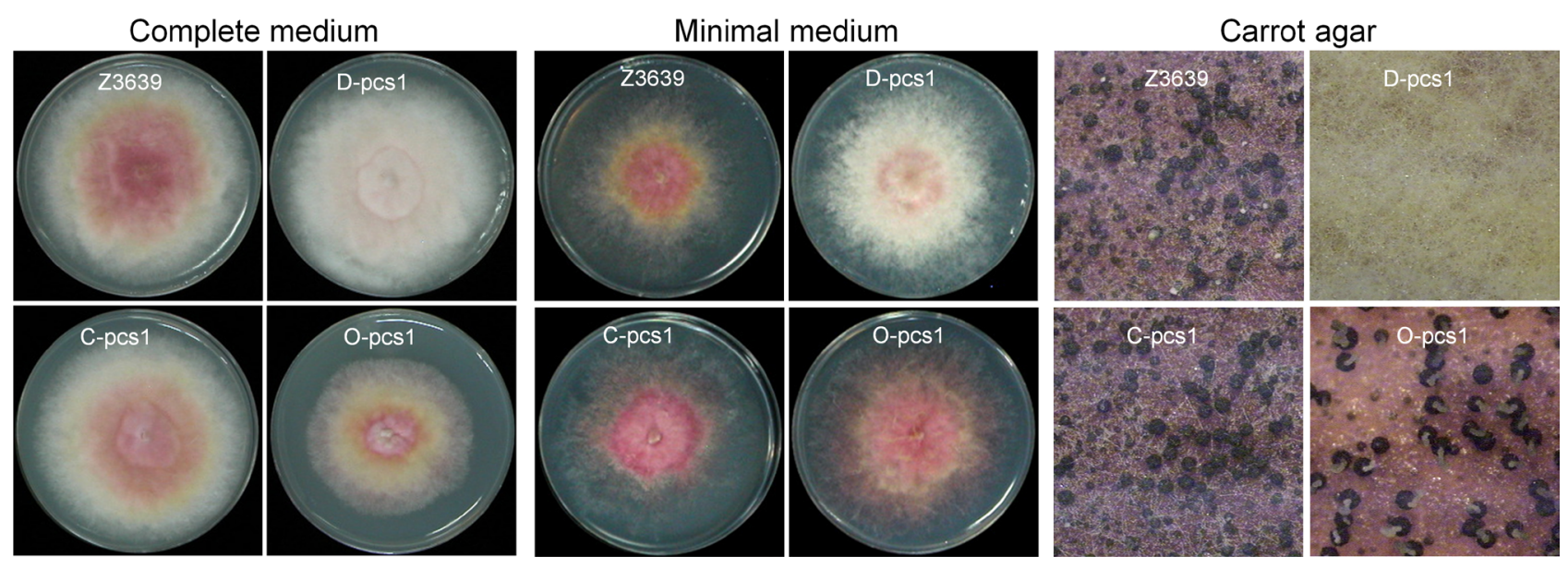

Fig. 1. Vegetative growth of G. zeae strains on CM and MM, and perithecia production on carrot agar. Z3639, wild-type strain; D-pcs1, pcs 1 deletion mutant; O-pcs1, mutant with constitutive expression of pcs1. Photos were taken after a 5-d incubation on CM and MM, and $7 \mathrm{~d}$ after sexual induction on carrot agar. 


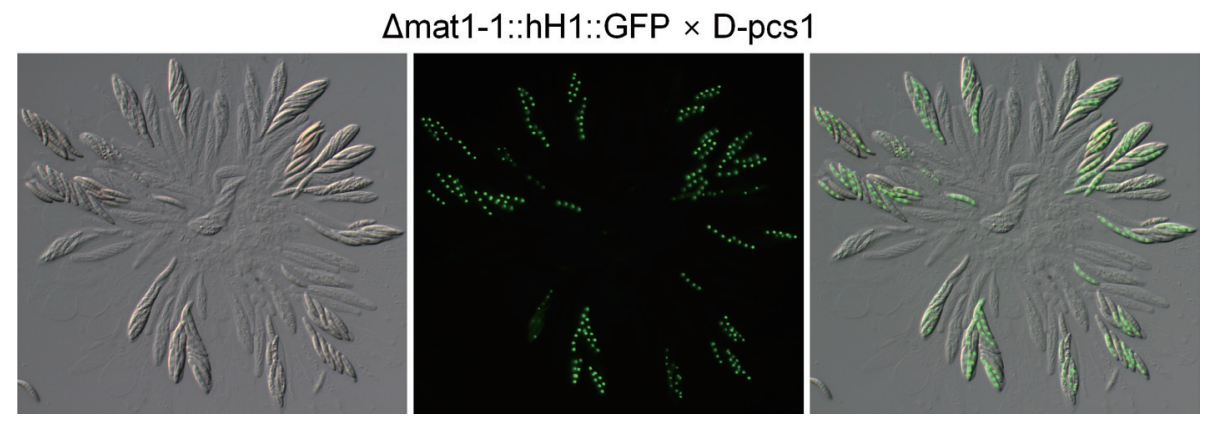

Fig. 2. Asci and ascospores produced by outcrossing between the heterothallic strain $\Delta m a t 1-1:: \mathrm{hH} 1:: \mathrm{GFP}$ and D-pcs1. Photos were taken in an 8-day incubation after sexual induction. Only four out of eight mature ascospores in each ascus fluoresced as observed by a fluorescent microscopy with a GFP filter. Two images, differential interference contrast (left) and GFP fluorescence (center), were merged (right).

into the $p c s 1$ deletion mutant (O-pcs1).

As described in a previous study (Son et al., 2011b), the $p s c 1$ deletion mutant (D-pcs1) produced less pigment on both CM and MM (Fig. 1) and lost the ability to selffertilize. In the D-pcs1 strain, the radial growth of mycelia on $\mathrm{MM}$, but not on $\mathrm{CM}$, was slightly increased compared to the wild-type strain (Table 2). In the C-pcs1 strain, all phenotypes observed in the D-pcs1 mutants were completely restored to wild-type phenotypes (Fig. 1 and Table
1), which confirmed that the phenotypic changes in D-pcs1 were triggered by the pcs 1 gene deletion. In O-pcs1, the strain with constitutive $p s c 1$ expression, the radial growth of mycelia on MM was not significantly different from the wild-type strain, but it was dramatically decreased on CM (Fig. 1 and Table 2).

For sexual development in the O-pcs1 strain, cirrhi were observed earlier than with the wild-type strain (Fig. 1). Although D-pcs1 lost self- and female fertility, it retained
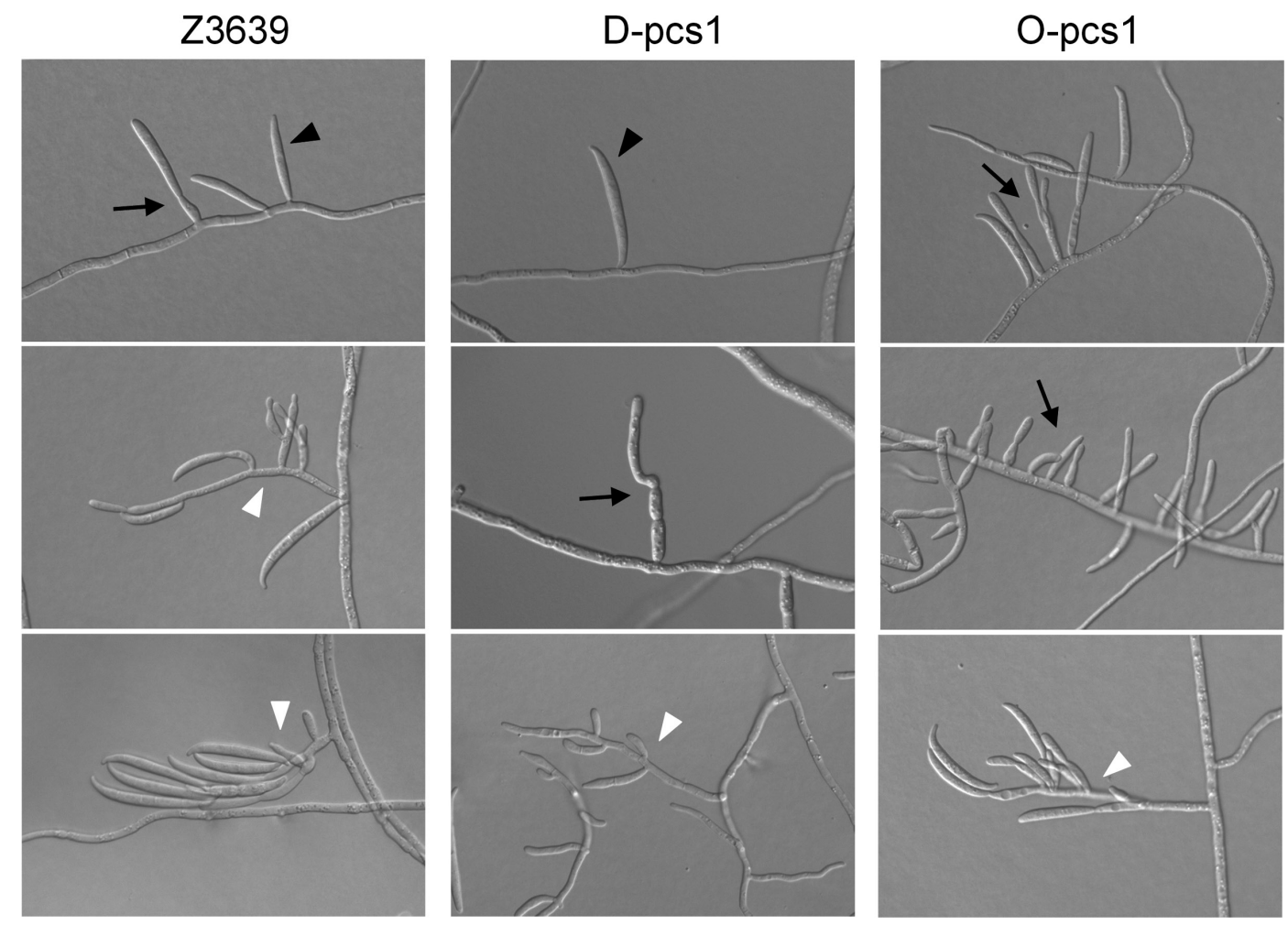

Fig. 3. Conidia production of $F$. graminearum strains on YMA. Black arrows indicate conidia produced from intercalary phialides on hyphae, and black arrowheads indicate conidia produced from intercalary phialides in false heads. White arrowheads indicate conidia produced from terminal phialides. 
male fertility, which resulted in normal sexual recombination when it was outcrossed (Fig. 2). The MIPS $F$. graminearum database (http://mips.helmholtz-muenchen. de/) reports that $p c s 1$ transcript gradually increases until 3 $\mathrm{d}$ after sexual induction. This expression profile combined with our results suggests that $p c s 1$ is responsible for earlier events of sexual reproduction.

Proper conidia production requires pcs1, which regulates conidia germination. In the submerged condition of CMC, D-pcs1 mutants showed a dramatic reduction in conidial production compared to the wild type and C-pcs1 (Table 2). The O-pcs1 strain produced approximately twofold more conidia compared to the wild type (Table 2). To verify conidia production in $F$. graminearum, we induced conidia production on YMA. In the wild-type strains, the majority of conidia were produced from terminal phialides on conidiophores and rarely produced from intercalary phialides on hyphae or intercalary phialides in false heads (Fig. 3). This observed conidia production in F. graminearum was similar to that described in $F$. oxysporum (Ohara and Tsuge, 2004), even though $F$. graminearum do not produce microconidia. In the D-pcs1 strain, terminal phialides produced less conidia than those of the wild-type strain, and conidia produced from intercalary phialides on hyphae often formed hyphae before conidiogenesis (Fig. 3). Additionally, often conidiation in conidiophores was arrested in the D-pcs1 strain, and conidiophores produced hyphae without conidiogenesis (Fig. 3). In the O-pcs1 strain, the majority of conidia were produced from intercalary phialides in false heads, and the number of intercalary

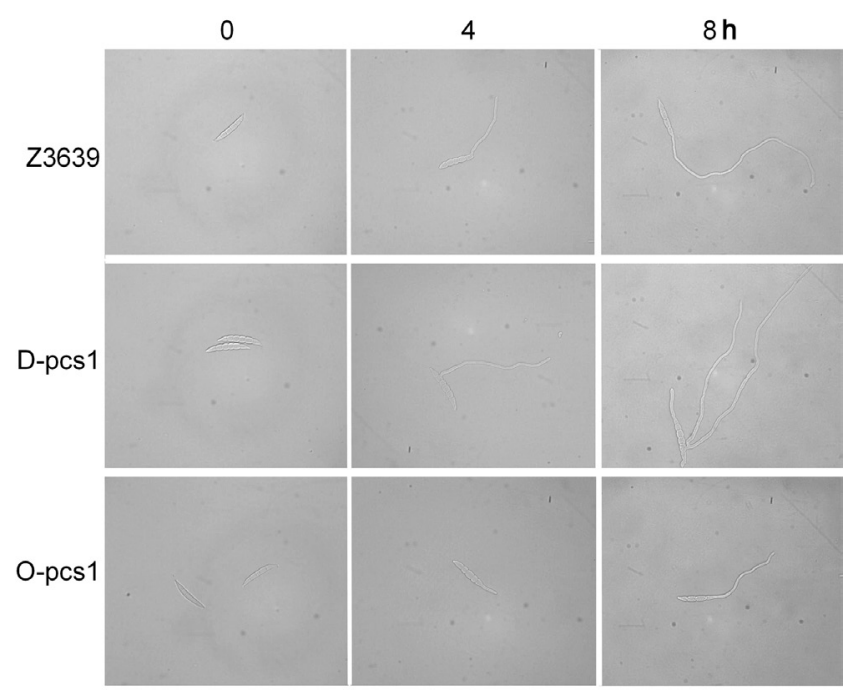

Fig. 4. Conidia germination after 0,4 , and $8 \mathrm{~h}$ incubation in minimal medium.

phialides on hyphae was dramatically increased compared to wild type (Fig. 3).

Conidia germination in the D-pcs1 mutants was faster than in the wild-type strain, while that of O-pcs1 was delayed compared to the wild-type strain (Fig. 4). The germination rate of conidia was not different between the wild type and D-pcs1, as in both strains approximately $80 \%$ of conidia were germinated after a 4 -h incubation, and $100 \%$ of conidia were germinated after an 8-h incubation. In contrast, only $30 \%$ and $70 \%$ of the O-pcs 1 conidia were germinated after 4- and 8-h incubations, respectively. The germination rate in each strain was not significantly differ-

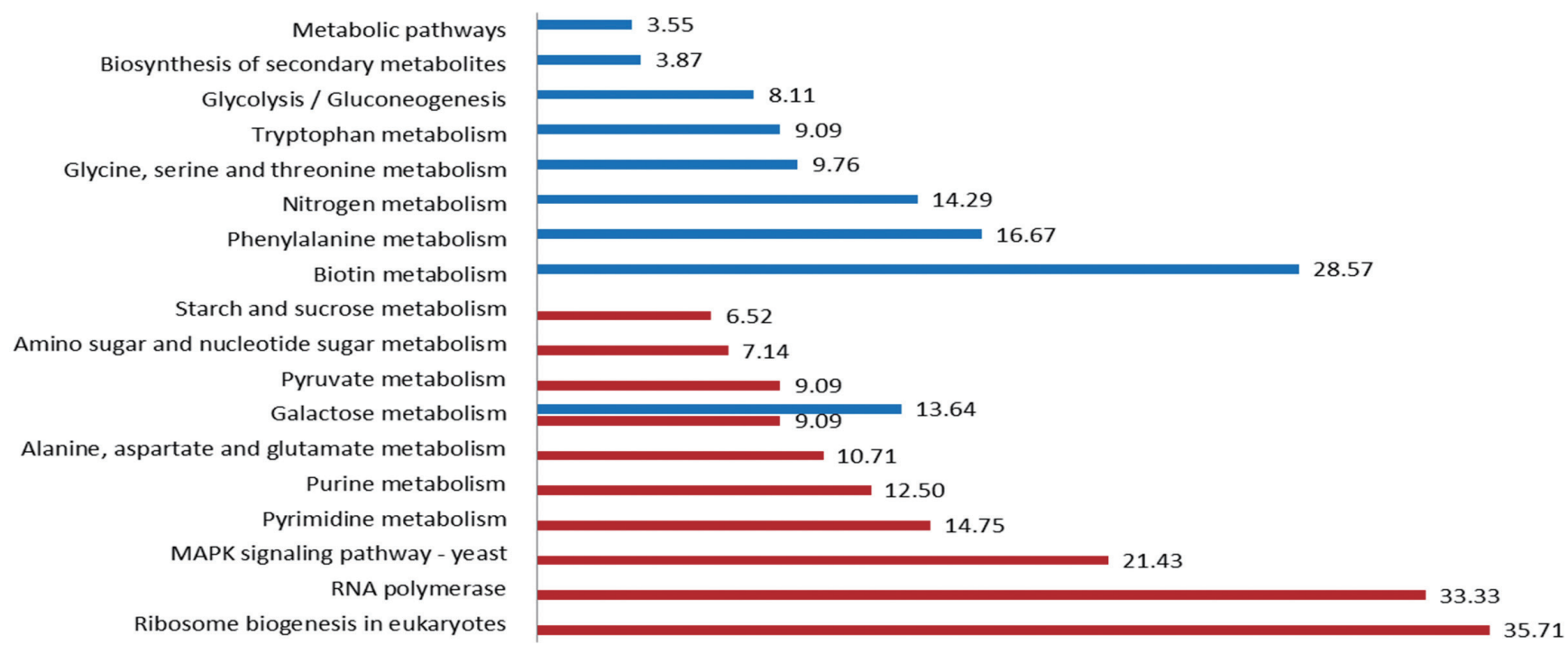

Fig. 5. Pathway enrichment of pcs1-dependent genes. Red and blue bars represent level of enrichment in positive and negative pcs 1 dependent genes, respectively. We set a cut-off with a $P$ value of $<0.05$. 
A

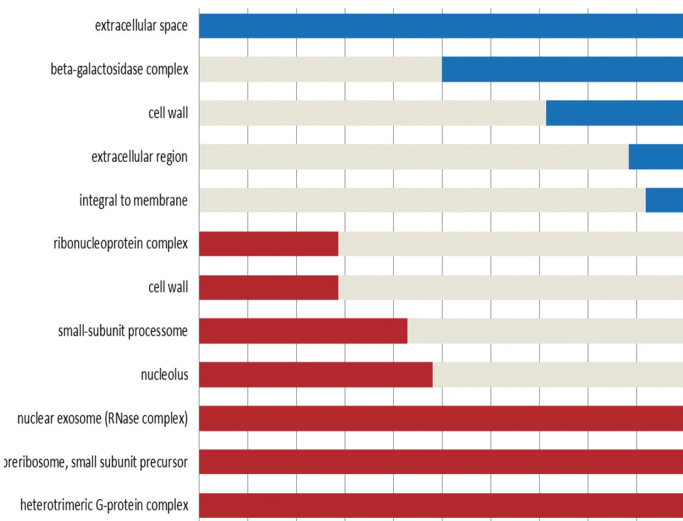

$\begin{array}{lllllllllll}0.00 & 10.00 & 20.00 & 30.00 & 40.00 & 50.00 & 60.00 & 70.00 & 80.00 & 90.00 & 100.00\end{array}$
B

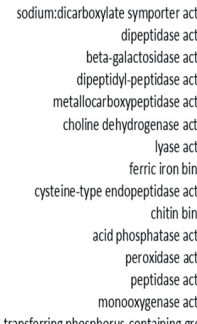

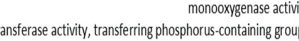
substrate-specific transmembrane transporter activity

C oxidoreductase activity, acting on paired donors, with incorporation or reduction of

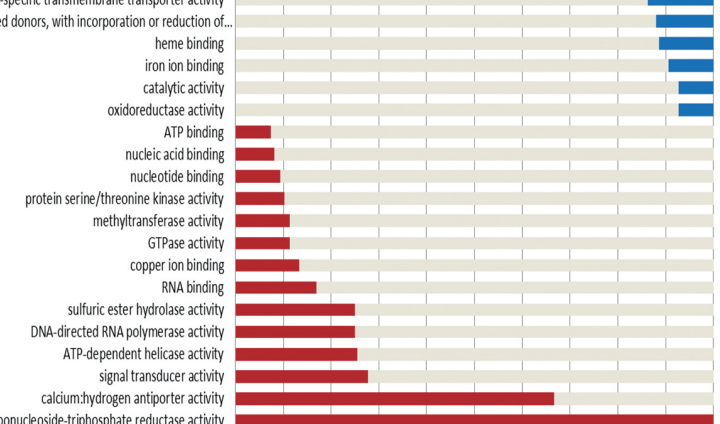

$\begin{array}{lllllllllll}0.00 & 10.00 & 20.00 & 30.00 & 40.00 & 50.00 & 60.00 & 70.00 & 80.00 & 90.00 & 100.0\end{array}$

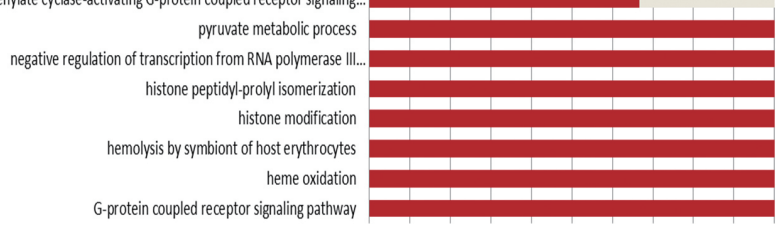

0.0010 .0020 .0030 .0040 .0050 .0060 .0070 .0080 .0090 .00100 .00

Fig. 6. Gene ontology (GO) enrichment of pcsl-dependent genes. (A) Cellular component, (B) biological process, (C) molecular function. Red and blue bars represent level of enrichment in positive and negative pcs l-dependent genes, respectively. We set a cut-off with a $P$ value of $<0.05$.

ent between $\mathrm{CM}$ and MM (data not shown).

Analysis of pcs1-dependent genes. Generally, the destruction of important transcription factors results in differential expression of genes involved in biological processes regulated by those transcription factors. Thus, to characterize $p c s 1$ function, we examined pcs 1 transcription factordependent genes, using both the KEGG pathway and GO. First, we selected differentially expressed genes that had greater than two-fold changes between D-pcs1 and wild type. We detected 1,280 pcs1-dependent genes out of a total of 11,624 genes. Enrichment analysis of KEGG pathways indicated that ribosome biogenesis, RNA polymerase, MAPK signaling pathway, and nucleotide metabolism are highly enriched in positively regulated $p c s 1$-dependent genes. Additionally, biotin, nitrogen, and several amino acid metabolisms are enriched in negatively regulated pcs1-dependent genes (Fig. 5). Furthermore, GO enrichment analysis indicated that G-protein signaling pathways in both cellular component and biological process, and 
RNA binding and processing in both biological process and molecular function are enriched in positively regulated pcs1-dependent genes (Fig. 6).

\section{Discussion}

In this study, we identified that $p c s 1$, a transcription factor, is responsible for conidia production, especially conidia generated from intercalary phialides on hyphae, and sexual reproduction. The observed overexpression of pcs 1 confirmed its role in the regulation of asexual and sexual reproduction.

In $\mathrm{CMC}$, conidia of the wild-type strain mainly were produced from intercalary phialides on hyphae, but terminal phialides and intercalary phialides in false heads were rarely observed (data not shown). Therefore, the dramatic reduction of D-pcs1 in conidia production in CMC may be triggered by inadequate generation of intercalary phialides and pcs 1 is especially required for intercalary phialides formation on hyphae. In addition, conidia germination in the D-pcs1 mutants was faster than in the wild-type strain, while that of O-pcs1 was delayed compared to the wildtype strain. These results suggest that $p c s 1$ also regulates conidia germination, and is partly responsible for the phase shift from conidia dormancy to germination.

Comparison of gene expression profiles between wild type and D-pcs1 revealed that pcs1 regulates genes involved in basic biological processes, such as RNA processing, ribosome biogenesis, and initial signaling cascades. Interestingly, we identified that $p c s 1$ positively regulates both asexual and sexual reproduction in $F$. graminearum. Particularly, G-protein signaling pathways in both cellular component and biological process are enriched. heterotrimeric $\mathrm{G}$ protein $\mathrm{G} \alpha$ subunit, $G z G P A 1$, responsible for sexual reproduction in F. graminearum (Yu et al., 2008), was highly down-regulated in D-pcs1 where mutants carrying deletion did not produce protoperithecia, indicating the loss of ability for sexual reproduction observed in Dpcs1 is closely related to down-regulation of $G z G P A 1$ gene in the mutant. Global regulators, velvet family proteins, are involved in diverse development stages including sexual and asexual reproduction. Under sexual development condition, velB deletion mutants do not produce protoperithecia but produce large numbers of conidia (Lee et al., 2012). However, velB expression was not changed in the D-pcs1 and $p c s 1$ gene expression was not affected by $v e l B$, indicating $p c s 1$ is independent of velB.

In conclusion, the $p c s 1$ transcription factor regulates genes involved in basic biological processes and initial signaling cascades such as G-protein signaling pathways as well as phenotypically regulates both conidiation and sexual reproduction in a positive manner and confers nutrient condition-dependent vegetative growth.

\section{Acknowledgments}

This work was supported by Basic Science Research Program through the National Research Foundation of Korea (NRF) funded by the Ministry of Science, ICT, and Future Planning (2012003883) and supported by grants from the Rural Development Administration (No. PJ009774).

\section{References}

Bowden, R. L. and Leslie, J. F. 1999. Sexual recombination in Gibberella zeae. Phytopathology 89:182-188.

Capellini, R. and Peterson, J. 1965. Macroconidium formation in submerged cultures by non-sporulating strains of Gibberella zeae. Mycologia 57:962-966.

Fernando, W. G. D., Paulitz, T. C., Seaman, W. L., Dutilleul, P. and Miller, J. D. 1997. Head blight gradients caused by Gibberella zeae from area sources of inoculums in wheat field plots. Phytopathology 87:414-421.

Guenther, J. C. and Trail, F. 2005. The development and differentiation of Gibberella zeae (anamorph: Fusarium graminearum) during colonization of wheat. Mycologia 97:229-237.

Hallen, H. E., Huebner, M., Shiu, S.-H., Güldener, U. and Trail, F. 2007. Gene expression shifts during perithecium development in Gibberella zeae (anamorph Fusarium graminearum), with particular emphasis on ion transport proteins. Fungal Genet. Biol. 44:1146-1156.

Han, Y.-K., Kim, M.-D., Lee, S.-H., Yun, S.-H. and Lee, Y.-W. 2007. A novel F-box protein involved in sexual development and pathogenesis in Gibberella zeae. Mol. Microbiol. 63:768779.

Harris, S. D. 2005. Morphogenesis in germinating Fusarium graminearum macroconidia. Mycologia 97:880-887.

Hong, S.-Y., So, J., Lee, J., Min, K., Son, H., Park, C., Yun, S.H. and Lee, Y.-W. 2010. Functional analyses of two syntaxinlike SNARE genes, GzSYN1 and GzSYN2, in the ascomycete Gibberella zeae. Fungal Genet. Biol. 47:364-372.

Horwitz, B. A., Sharon, A., Lu, S.-W., Ritter, V., Sandrock, T. M., Yoder, O. C. and Turgeon, B. G. 1999. A G protein alpha subunit from Cochliobolus heterostrophus involved in mating and appressorium formation. Fungal Genet. Biol. 26:19-32.

Hou, Z., Xue, C., Peng, Y., Katan, T., Kistler, H. C. and Xu, J.R. 2002. A mitogen-activated protein kinase gene (MGV1) in Fusarium graminearum is required for female fertility, heterokaryon formation, and plant infection. Mol. PlantMicrobe Interact. 15:1119-1127.

Lee, J., Chang, I.-Y., Kim, H., Yun, S.-H., Leslie, J. F. and Lee, Y.-W. 2009. Genetic diversity and fitness of Fusarium 
graminearum populations from rice in Korea. Appl. Environ. Microbiol. 75:3289-3295.

Lee, J., Kim, H., Jeon, J.-J., Kim, H.-S., Zeller, K. A., Carter, L. L. A., Leslie, J. F. and Lee, Y.-W. 2012. Population structure of and mycotoxin production by Fusarium graminearum from maize in South Korea. Appl. Environ. Microbiol. 78:21612167.

Lee, J., Lee, T., Lee, Y.-W., Yun, S.-H. and Turgeon, B. G. 2003. Shifting fungal reproductive mode by manipulation of mating type genes: obligatory heterothallism of Gibberella zeae. Mol. Microbiol. 50:145-152.

Lee, J., Leslie, J. F. and Bowden, R. L. 2008. Expression and function of sex pheromones and receptors in the homothallic ascomycete Gibberella zeae. Eukaryot. Cell 7:1211-1221.

Lee, J., Myong, K., Kim, J.-E., Kim, H.-K., Yun, S.-H. and Lee, Y.-W. 2012. FgVelB globally regulates sexual reproduction, mycotoxin production and pathogenicity in the cereal pathogen Fusarium graminearum. Microbiology 158:17231733.

Lee, J., Park, C., Kim, J.-C., Kim, J. E. and Lee, Y.-W. 2010. Identification and functional characterization of genes involved in the sexual reproduction of the ascomycete fungus Gibberella zeae. Biochem. Biophys. Res. Commun. 401:4852.

Lee, S. H., Lee, J., Lee, S., Park, E.-H., Kim, K.-W., Kim, M.D., Yun, S.-H. and Lee, Y.-W. 2009. GzSNF1 is required for normal sexual and asexual development in the ascomycete Gibberella zeae. Eukaryot. Cell 8:116-127.

Lee, S., Son, H., Lee, J., Min, G., Choi, K. J., Kim, J.-C. and Lee, Y.-W. 2011. Functional analyses of two acetyl coenzyme A synthetases in the ascomycete Gibberella zeae. Eukaryot. Cell 10:1043-1052.

Leslie, J. F. and Summerell, B. A. 2006. The Fusarium laboratory manual. Blackwell Professional, Ames, IA, USA.

Lin, Y., Son, H., Lee, J., Min, K., Choi, G. J., Kim, J.-C. and Lee, Y.-W. 2011. A putative transcription factor MYT1 is required for female fertility in the ascomycete Gibberella zeae. PLoS ONE 6:e25586.

Min, K., Lee, J., Kim, J.-C., Kim, S. G., Kim, Y. H., Vogel, S., Trail, F. and Lee, Y.-W. 2010. A novel gene, $R O A$, is required for normal morphogenesis and discharge of ascospores in Gibberella zeae. Eukaryot. Cell 9:1495-1503.

Ohara, T. and Tsuge, T. 2004. FoSTUA, encoding a basin helixloop-helix protein, differentially regulates development of three kinds of asexual spores, macroconidia, microconidia, and chlamydospores, in the fungal plant pathogen Fusarium oxysporum. Eukaryot. Cell 3:1412-1422.

Qi, W., Kwon, C. and Trail, F. 2006. Microarray analysis of transcript accumulation during perithecium development in the filamentous fungus Gibberella zeae (anamorph Fusarium graminearum). Mol. Genet. Genomics 276:87-100.

Sambrook, J. and Russell, D. W. 2001. Molecular cloning: a laboratory manual. Cold Spring Harbor Laboratory Press, Cold Spring Harbor, NY, USA.

Seong, K.-Y., Zhao, X., Xu, J.-R., Güldener, U. and Kostler, H. C. 2008. Conidial germination in the filamentous fungus Fusarium graminearum. Fungal Genet. Biol. 45:389-399.

Son, H., Lee, J., Park, A. R. and Lee, Y.-W. 2011a. ATP citrate lyase is required for normal sexual and asexual development in Gibberella zeae. Fungal Genet. Biol. 48:408-417.

Son, H., Seo, Y.-S., Min, K., Park, A. R., Lee, J., Jin, J. M., Lin, Y., Cao, P., Hong, S.-Y., Kim, E. K. et al. 2011b. A phenomebased functional analysis of transcription factors in the cereal head blight fungus, Fusarium graminearum. PLoS Pathog. 7:e1002310.

Yu, H.-Y., Seo, J.-A., Kim, J.-E., Han, K.-H., Shim, W.-B., Yun, S.-H. and Lee, Y.-W. 2008. Functional analyses of heterotrimeric $\mathrm{G}$ protein $\mathrm{G} \alpha$ and $\mathrm{G} \beta$ subunits in Gibberella zeae. Microbiology 154:392-401.

Yu, J.-H., Hamari, Z., Han, K.-H., Seo, J.-A., Reyes-Dominguez, Y. and Scazzocchio, C. 2004. Double-joint PCR: a PCRbased molecular tool for gene manipulations in filamentous fungi. Fungal Genet. Biol. 41:973-981.

Sutton, J. C. 1982. Epidemiology of wheat head blight and maize ear rot caused by Fusarium graminearum. Can. J. Plant Pathol. 4:195-209.

Zeller, K. A., Bowden, R. L. and Leslie, J. F. 2003. Diversity of epidemic populations of Gibberella zeae from small quadrats in Kansas and North Dakota. Phytopathology 93:874-880.

Zeller, K. A., Bowden, R. L. and Leslie, J. F. 2004. Population differentiation and recombination in wheat scab populations of Gibberella zeae from the United States. Mol. Ecol. 13:563-571.

Zenczmionka, N. J., Maier, F. J., Lösch, A. P. and Schäfer, W. 2003. Mating, conidiation and pathogenicity of Fusarium graminearum, the main causal agent of the head-blight disease of wheat, are regulated by the MAP kinase gpmk1. Curr. Genet. 43:87-95. 